\title{
Middle Cerebral Artery Pseudoaneurysm Formation Following Stereotactic Biopsy
}

\author{
Mahmoud Rayes, Diaa A. Bahgat, William J. Kupsky, Sandeep Mittal
}

Can. J. Neurol. Sci. 2009; 35: 664-668

Trauma accounts for less than $1 \%$ of all intracranial aneurysms. ${ }^{1}$ Based on the underlying mechanism of injury, traumatic intracranial aneurysms (TICA) can be classified into four categories: penetrating head injury, missile injury, blunt head injury, and iatrogenic injury. ${ }^{1-3}$ Histologically, there are two types of TICAs: true and false. The majority of TICAs are false aneurysms and are also referred to as pseudoaneurysms., ${ }^{2,4}$ Establishing a diagnosis of pseudoaneurysms remains difficult and requires a high index of suspicion. In the absence of timely diagnosis and treatment, a mortality rate of up to $50 \%$ has been reported in patients harboring a cerebral pseudoaneurysm. ${ }^{3} \mathrm{We}$ present a case of distal middle cerebral artery (MCA) pseudoaneurysm following a stereotactic needle biopsy of a right temporal lobe mass. To our knowledge, only one other report in the literature describes the formation of pseudoaneurysm following stereotactic brain biopsy. Our case is the only reported case where the diagnosis of iatrogenic pseudoaneurysm following stereotactic biopsy was made prior to definitive surgery.

\section{Case Presentation}

History. A 22-year-old left-handed Caucasian male presented with a history of ongoing slurring of speech, decreased coordination, and increased inhibition. Magnetic resonance imaging of the brain revealed an enhancing lesion extending from the right temporal lobe into the basal ganglia (Figure 1). An image-guided stereotactic needle biopsy of the lesion was undertaken at an outside institution. No unexpected bleeding was encountered. Histopathology was in keeping with a primary CNS lymphoma. The patient was treated with high-dose methotrexate and received eight cycles over a three month period with excellent response. Residual hemorrhage was seen over the right temporal cortex at the biopsy site on the follow-up MRI done three months after the biopsy (Figure 2). One month later, MRI showed regression of the lymphoma and decrease in the residual hemorrhage at the biopsy site. Six months later, MRI showed complete resolution of the residual parenchymal hemorrhage. However, a rounded contrast-enhancing lesion measuring $9.4 \mathrm{x}$ $8.6 \mathrm{~mm}$ related to the nearby cortical vessels was first noticed (Figure 3A). The concern at that time was that this new area of enhancement represented recurrence of the tumor.

Neuroimaging. A follow-up MRI obtained six months later showed an increase in the size of the lesion to $11.9 \times 12.7 \mathrm{~mm}$ (Figures 3B-C). The patient remained asymptomatic and his primary CNS lymphoma was in remission. At this time, a traumatic pseudoaneurysm was suspected. The patient was therefore referred to us for further evaluation. An MR angiogram showed an aneurysmal dilatation of the distal segment of the right MCA. Digital substraction angiography confirmed the presence of a pseudoaneurysm related to two cortical M4 branches (Figure 4). Aggressive treatment of the iatrogenic false traumatic aneurysm was recommended because of the increasing size and inherent risk of rupture. Endovascular treatment was felt to be suboptimal because of its distal location. Therefore, a microsurgical approach was chosen.

Surgery. A $4 \mathrm{~cm}$ right temporal bone flap, centered over the previous biopsy site, was elevated. The dura was carefully dissected and the pseudoaneurysm was identified on the cortical surface just underneath the gliotic tissue of the original burr hole. Hemosiderin staining was evident in the region. The

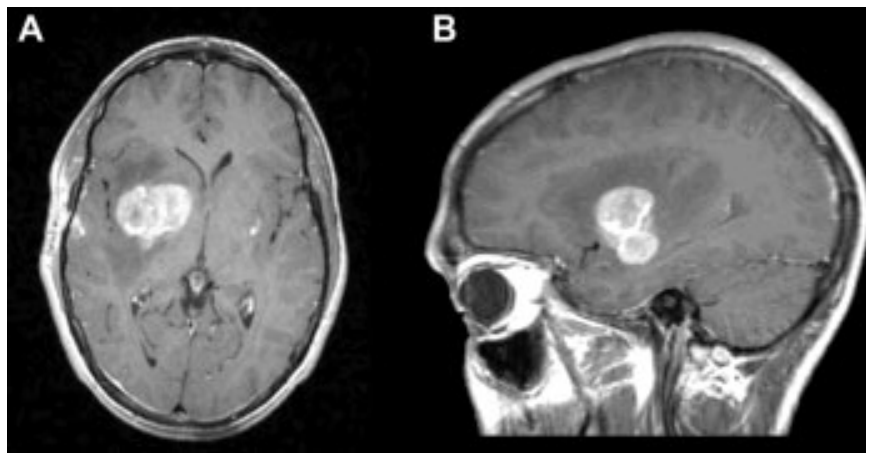

Figure 1: Axial (A) and sagittal (B) T1-weighted MRI scan demonstrating a large heterogeneous enhancing mass in the right basal ganglia region extending to the right temporal lobe.

From the Departments of Neurosurgery and Neuropathology, Karmanos Cancer Institute, Wayne State University, Detroit, Michigan, USA.

Received March 26, 2008. Final Revisions Submitted June 13, 2008.

Correspondence to: Sandeep Mittal, Dept. of Neurosurgery, Wayne State University, 4160 John R Street, Suite 930, Detroit, Michigan, 48201, USA. 


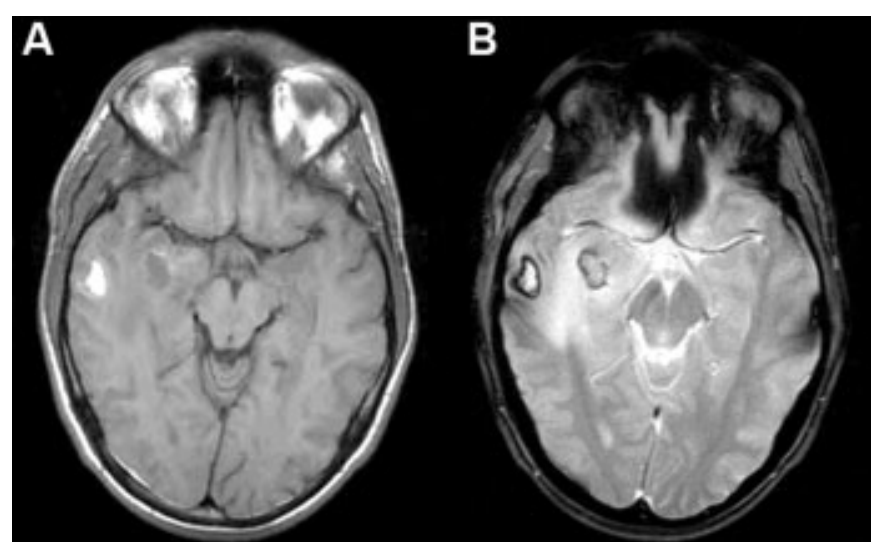

Figure 2: Postoperative axial T1 without contrast (A) and T2-weighted (B) scans showing hyperintense areas in the right temporal related to blood products. Note the hemosiderin rim around the small hematoma.

pseudoaneurysm was circumferentially dissected free of adhesions. The parent artery was a M4 branch which bifurcated into two smaller branches distal to the pseudoaneurysm (Figure $5 \mathrm{~A})$. The pseudoaneurysm was secured by applying small temporary aneurysm clips to the feeding artery as well as the distal branches. The false aneurysm was resected and end-to-end microanastomosis of the proximal and distal MCA segments was performed using 8-0 Prolene sutures. We elected to proceed with reanastomosis of the distal branches to the parent vessel rather than simple trapping of the pseudoaneurysm because of the large caliber of the distal MCA branches and lack of collateral supply. Adequate blood flow in the distal branches was confirmed with intraoperative Doppler ultrasonography.

Histology and Postoperative Course. Histopathological examination of the excised pseudoaneurysm revealed partial occlusion of the lumen by an organizing thrombus (Figure 5B). The wall of the false aneurysm consisted of fibrous tissue with absent smooth muscle and internal elastic lamina. The patient tolerated the procedure well without any complications or deficits. He was discharged from the hospital two days later in stable condition.

\section{DISCUSSION}

Traumatic intracranial aneurysms are rare and account for less than $1 \%$ of all intracranial aneurysms. ${ }^{1,6,7}$ They can occur following penetrating as well as blunt head injury. ${ }^{8-12}$ However, penetrating head injury is a more common cause of TICA formation than blunt head injury. ${ }^{2,12-15}$ Iatrogenic trauma has also been reported as a cause for TICAs. In a recent review, 52 cases of iatrogenic intracranial aneurysms were described in the literature since $1955 .{ }^{16}$ They commonly involved the internal carotid artery and rarely the middle cerebral artery. ${ }^{16}$ Pseudoaneurysms have been reported to occur following insertion of ventriculo-peritoneal shunts and intracranial pressure monitors, aneurysm surgery, tumor surgery, endoscopic ventriculostomy, and transsphenoidal surgery. ${ }^{16-23}$

Histologically, traumatic intracranial aneurysms are classified as true or false. True aneurysms are formed after partial disruption of the arterial wall. Thus, the internal elastic lamina and media are damaged, while only the adventitia and, possibly, a thinned or scarred layer of media remains intact. Incomplete mural injury with subsequent dilatation of intact but weakened arterial wall forms a true aneurysm. On the other hand, a false aneurysm is formed after complete arterial wall disruption, often sealed by a hematoma. The hematoma, released after the arterial injury, organizes to form the outer wall of the pseudoaneurysm. The majority of TICAs are pseudoaneurysms., ${ }^{2,4,5}$ There are two types of iatrogenic pseudoaneurysms: saccular and fusiform. ${ }^{24}$ Fusiform pseudoaneurysms are typically caused by peeling of the tumor capsule from the arterial adventitia of the adjacent vessel during the surgery. ${ }^{24}$ These generally do not enlarge with time and typically do not present with a high incidence of hemorrhage. In contrast, saccular aneurysms result from a more focal and complete laceration of the vessel wall. They have a greater tendency to rupture in comparison to fusiform pseudoaneurysms. ${ }^{16}$ Unlike berry aneurysms, traumatic intracranial aneurysms typically do not have a neck, are more irregular in their dome contour, and are subject to delayed filling and emptying of the sac when viewed on cerebral angiograms. ${ }^{14}$

The natural history of p s e u d o a n e u r y s m s suggests a relatively dire course..$^{2,13,14,25}$ The time
Figure 3: Serial axial T1-weighted enhanced MRI scans ( $A$ and B) demonstrating resolution of the hematoma and appearance of an enlarging round enhancing lesion in the superficial aspect of the right mid-temporal lobe. Axial T2-weighted image $(C)$ showing the hemosiderin rim related to prior hemorrhage at the site. 

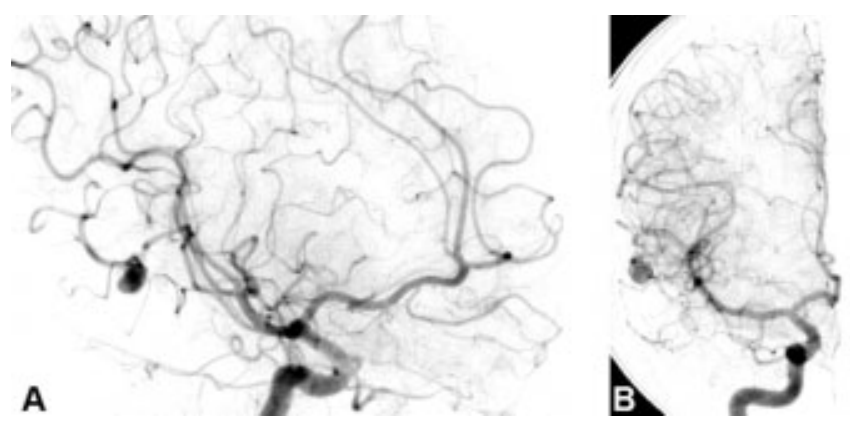

Figure 4: Cerebral angiogram with lateral oblique (A) and $A-P(B)$ views showing an irregular aneurysm involving the distal right middle cerebral artery.

from injury to diagnosis of TICAs varies from several hours to several months, and even several years in some cases..$^{26-29}$ Unfortunately, the majority of cases are diagnosed after rupture. Spontaneous resolution or shrinkage has also been reported in the literature ${ }^{6,30-32}$ In one series, shrinkage was seen in nearly $20 \%$ of patients with TICAs on serial angiography. ${ }^{30}$ Horiuchi and colleagues ${ }^{14}$ reported a rupture rate of $72.4 \%$ in their report of traumatic MCA aneurysms. Pseudoaneurysms composed $83.3 \%$ of all TICAs in their review. They reported a favorable outcome in $75 \%$ of patients who were treated with surgery and in $37.5 \%$ of patients managed conservatively. ${ }^{14}$ Furthermore, the natural history of TICAs tends to be quite unfavorable as the mortality rate has been reported to be as high as $50 \%{ }^{3,33}$ Thus, aggressive management of traumatic saccular pseudoaneurysms is generally recommended in order to avoid the elevated mortality rate and achieve a more favorable outcome. . $^{3,13,14,33}$

Despite it's minimally invasive nature, image-guided stereotactic brain biopsy inherently carries risk of transient neurological deficit, seizure, infection, and hemorrhage. ${ }^{34,35}$ The reported incidence of surgical complications varies between $0-9.3 \% .^{34,36-38} \mathrm{~A}$ meta-analysis of almost 5000 published cases of CT-guided stereotactic biopsy of intracranial lesions revealed an overall complication rate of approximately 5\%.34 The most common complication encountered is hemorrhage at the biopsy site or along the needle tract. ${ }^{34,39}$ In a series of 355 patients, Grossman and coworkers ${ }^{39}$ reported a $3.4 \%$ and $3.6 \%$ incidence of asymptomatic and symptomatic hemorrhagic complications, respectively following diagnostic stereotactic-guided biopsy. ${ }^{39}$ Nevertheless, the incidence of pseudoaneurysm formation following stereotactic needle biopsy remains unknown.

Our review of the literature revealed only one other case describing the formation of a pseudoaneurysm following a stereotactic-guided brain biopsy. Sahrakar and colleagues ${ }^{35}$ reported the case of a three-year-old child who underwent stereotactic biopsy for a frontal cystic lesion. Histology confirmed a well-differentiated astrocytoma and definitive surgical resection was subsequently undertaken. A pseudoaneurysm, contiguous with a cortical artery, was incidentally discovered during the second surgery. Anterograde and retrograde blood flow was confirmed in the parent vessel before ligation of the pseudoaneurysm which was coagulated and subsequently resected with a portion of the parent vessel. The authors recommended direct visualization of the cortex with an appropriately sized burr hole to avoid passing the needle through a cortical sulcus and thereby help to avoid arterial injury during image-guided brain biopsies. ${ }^{35}$

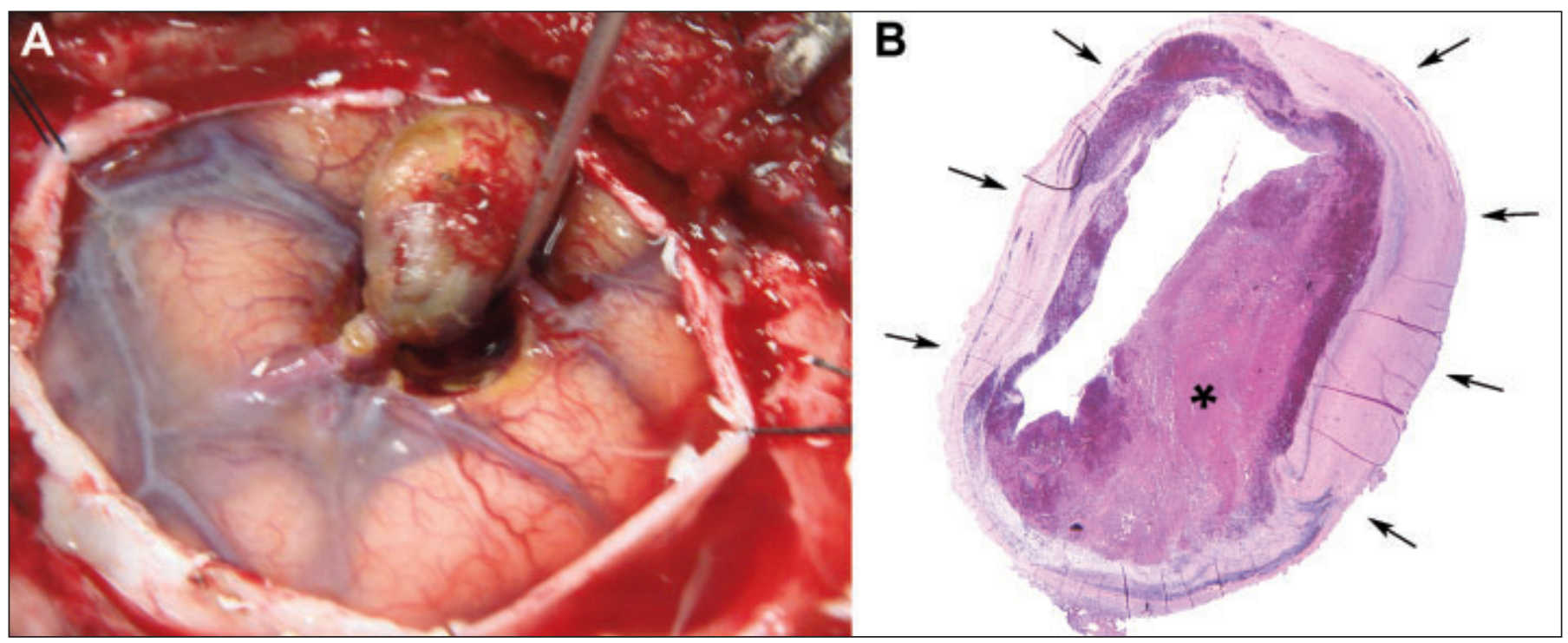

Figure 5: Intraoperative view of the right MCA pseudoaneurysm (A) showing the feeding artery and one of the two distal branches. Photomicrograph of the pseudoaneurysm (B). Hematoxylin and eosin staining demonstrating that the lumen is partly occluded by organizing thrombus $(*)$. The vessel wall consists of fibrous tissue and old organized thrombus without smooth muscle or elastica (arrows). Original magnification, x20. 
In our case, the pseudoaneurysm was noted following stereotactic-guided biopsy for a right temporal lesion. The distal MCA traumatic aneurysm was followed with serial MRIs and showed evidence of enlargement. The diagnosis was confirmed before surgery on MRA and cerebral angiography. Endovascular treatment was not recommended because of the distal location of the pseudoaneurysm. Thus, surgical trapping and excision was undertaken. The pseudoaneurysm was secured, resected, and primary vessel reconstruction with anastomosis of the proximal and distal MCA segments was accomplished.

The diagnosis of pseudoaneurysms can be quite challenging. Treatment modalities include direct aneurysmal clipping, trapping or occlusion of the proximal parent, and endovascular coiling or occlusion. ${ }^{1,2,21,40-42}$ However, surgical clipping may not be suitable in many instances because pseudoaneurysms do not have a neck on which the clip can be secured. ${ }^{2}$ Furthermore, endovascular treatment can be challenging because pseudoaneurysms may occur at any segment of the vessel and not necessarily at a branching point. In addition, they typically have a high neck to fundus ratio which may make them less suitable for endovascular management. ${ }^{3,20}$ Surgical trapping and excision is usually recommended for distal traumatic MCA aneurysms. On the other hand, more proximal pseudoaneurysms can be adequately treated by clipping, trapping with bypass, or endovascular techniques to preserve distal blood flow. ${ }^{14}$

Pseudoaneurysms are rare pathological entities and often remain undiagnosed until time of rupture. We present the second case in the literature which describes the formation of a pseudoaneurysm following image-guided stereotactic brain biopsy. Aggressive management is recommended because of the unfavorable natural history and high mortality rate associated with traumatic pseudoaneurysms. The optimal modality of treatment, either microsurgical or endovascular, should be determined on a case by case scenario.

\section{REFERENCES}

1. Uzan M, Cantasdemir M, Seckin MS, Hanci M, Kocer N, Sarioglu $\mathrm{AC}$, et al. Traumatic intracranial carotid tree aneurysms. Neurosurgery. 1998; Dec 43(6):1314-20.

2. Amirjamshidi A, Rahmat H, Abbassioun K. Traumatic aneurysms and arteriovenous fistulas of intracranial vessels associated with penetrating head injuries occurring during war: principles and pitfalls in diagnosis and management. A survey of 31 cases and review of the literature. J Neurosurg. 1996; May 84(5):769-80.

3. Larson PS, Reisner A, Morassutti DJ, Abdulhadi B, Harpring JE. Traumatic intracranial aneurysms. Neurosurg Focus. 2000; 8(1):e4.

4. Hachemi M, Jourdan C, Di Roio C, Turjman F, Ricci-Franchi A, Mottolese C, et al. Delayed rupture of traumatic aneurysm after civilian craniocerebral gunshot injury in children. Childs Nerv Syst. 2007; Mar 23(3):283-7.

5. Horowitz MB, Kopitnik TA, Landreneau F, Ramnani DM, Rushing EJ, George E, et al. Multidisciplinary approach to traumatic intracranial aneurysms secondary to shotgun and handgun wounds. Surg Neurol. 1999; Jan 51(1):31-41.

6. Benoit BG, Wortzman G. Traumatic cerebral aneurysms. Clinical features and natural history. J Neurol Neurosurg Psychiatry. 1973; Feb 36(1):127-38.

7. Fleischer AS, Patton JM, Tindall GT. Cerebral aneurysms of traumatic origin. Surg Neurol. 1975; Aug 4(2):233-9.

8. Saito K, Baskaya MK, Shibuya M, Suzuki Y, Sugita K. False traumatic aneurysm of the dorsal wall of the supraclinoid internal carotid artery--case report. Neurol Med Chir (Tokyo). 1995; Dec 35(12):886-91.
9. Nishioka T, Maeda Y, Tomogane Y, Nakano A, Arita N. Unexpected delayed rupture of the vertebral-posterior inferior cerebellar artery aneurysms following closed head injury. Acta Neurochir (Wien). 2002; Aug 144(8):839-45.

10. Lath R, Vaniprasad A, Kat E, Brophy BP. Traumatic aneurysm of the callosomarginal artery. J Clin Neurosci. 2002; Jul 9(4):466-8.

11. Komiyama M, Morikawa T, Nakajima H, Yasui T, Kan M. "Early" apoplexy due to traumatic intracranial aneurysm--case report. Neurol Med Chir (Tokyo). 2001; May 41(5):264-70.

12. Murakami M, Kakita K, Hosokawa Y. Ruptured traumatic aneurysm after trivial injury mimicking acute spontaneous subdural hematoma--case report. Neurol Med Chir (Tokyo). 2003; Mar 43(3):130-3.

13. Chedid MK, Vender JR, Harrison SJ, McDonnell DE. Delayed appearance of a traumatic intracranial aneurysm. Case report and review of the literature. J Neurosurg. 2001; Apr 94(4):637-41.

14. Horiuchi T, Nakagawa F, Miyatake M, Iwashita T, Tanaka Y, Hongo $\mathrm{K}$. Traumatic middle cerebral artery aneurysm: case report and review of the literature. Neurosurg Rev. 2007; Jul 30(3):263-7.

15. Ohta M, Matsuno H. Proximal M2 false aneurysm after head trauma--Case report. Neurol Med Chir (Tokyo). 2001; Mar 41(3):131-4.

16. Ciceri EF, Regna-Gladin C, Erbetta A, Chiapparini L, Nappini S, Savoiardo M, et al. Iatrogenic intracranial pseudoaneurysms: neuroradiological and therapeutical considerations, including endovascular options. Neurol Sci. 2006; Nov 27(5):317-22.

17. Shirane R, Kondo T, Yoshida YK, Furuta S, Yoshimoto T. Ruptured cerebral pseudoaneurysm caused by the removal of a ventricular catheter. Case report. J Neurosurg. 1999; Dec 91(6):1031-3.

18. Le H, Munshi I, Macdonald RL, Wollmann R, Frank J. Traumatic aneurysm resulting from insertion of an intracranial pressure monitor. Case illustration. J Neurosurg. 2001; Oct 95(4):720.

19. Jenkinson MD, Basu S, Broome JC, Eldridge PR, Buxton N. Traumatic cerebral aneurysm formation following ventriculoperitoneal shunt insertion. Childs Nerv Syst. 2006; Feb 22(2):193-6.

20. Cappabianca P, Briganti F, Cavallo LM, de Divitiis E. Pseudoaneurysm of the intracavernous carotid artery following endoscopic endonasal transsphenoidal surgery, treated by endovascular approach. Acta Neurochir (Wien). 2001;143(1):95-6.

21. Tokunaga K, Kusaka N, Nakashima H, Date I, Ohmoto T. Coil embolization of intradural pseudoaneurysms caused by arterial injury during surgery: report of two cases. AJNR Am J Neuroradiol. 2001; Jan 22(1):35-9.

22. McLaughlin MR, Wahlig JB, Kaufmann AM, Albright AL. Traumatic basilar aneurysm after endoscopic third ventriculostomy: case report. Neurosurgery. 1997; Dec 41(6): 1400-3; discussion 3-4.

23. Dunn IF, Woodworth GF, Siddiqui AH, Smith ER, Vates GE, Day AL, et al. Traumatic pericallosal artery aneurysm: a rare complication of transcallosal surgery. Case report. J Neurosurg. 2007; Feb 106: Suppl 2:153-7.

24. Sutton LN. Vascular complications of surgery for craniopharyngioma and hypothalamic glioma. Pediatr Neurosurg. 1994; 21 Suppl 1:124-8.

25. Bruneau M, Gustin T, Zekhnini K, Gilliard C. Traumatic false aneurysm of the middle meningeal artery causing an intracerebral hemorrhage: case report and literature review. Surg Neurol. 2002; Mar 57(3):174-8.

26. Aarabi B. Traumatic aneurysms of brain due to high velocity missile head wounds. Neurosurgery. 1988; Jun 22(6 Pt 1):105663.

27. Haddad FS, Haddad GF, Taha J. Traumatic intracranial aneurysms caused by missiles: their presentation and management. Neurosurgery. 1991; Jan 28(1):1-7.

28. McDonald EJ, Winestock DP, Hoff JT. The value of repeat cerebral arteriography in the evaluation of trauma. AJR Am J Roentgenol. 1976; Apr 126(4):792-7.

29. Salar G, Mingrino S. Traumatic intracranial internal carotid aneurysm due to gunshot wound. Case report. J Neurosurg. 1978; Jul 49(1):100-2.

30. Parkinson D, West M. Traumatic intracranial aneurysms. J Neurosurg. 1980; Jan 52(1):11-20. 
31. Rumbaugh CL, Bergeron RT, Talalla A, Kurze T. Traumatic aneurysms of the cortical cerebral arteries. Radiographic aspects. Radiology. 1970; Jul 96(1):49-54.

32. Shah Q, Friedman J, Mamourian A. Spontaneous resolution of traumatic pseudoaneurysm of the middle meningeal artery. AJNR Am J Neuroradiol. 2005; Nov-Dec 26(10):2530-2.

33. Holmes B, Harbaugh RE. Traumatic intracranial aneurysms: a contemporary review. J Trauma. 1993; Dec 35(6):855-60.

34. Bernstein M, Parrent AG. Complications of CT-guided stereotactic biopsy of intra-axial brain lesions. J Neurosurg. 1994; Aug 81(2):165-8.

35. Sahrakar K, Boggan JE, Salamat MS. Traumatic aneurysm: a complication of stereotactic brain biopsy: case report. Neurosurgery. 1995; Apr 36(4):842-6.

36. Kulkarni AV, Guha A, Lozano A, Bernstein M. Incidence of silent hemorrhage and delayed deterioration after stereotactic brain biopsy. J Neurosurg. 1998; Jul 89(1):31-5.

37. Field M, Witham TF, Flickinger JC, Kondziolka D, Lunsford LD. Comprehensive assessment of hemorrhage risks and outcomes after stereotactic brain biopsy. J Neurosurg. 2001; Apr 94(4): $545-51$.
38. McGirt MJ, Woodworth GF, Coon AL, Frazier JM, Amundson E, Garonzik I, et al. Independent predictors of morbidity after image-guided stereotactic brain biopsy: a risk assessment of 270 cases. J Neurosurg. 2005; May 102(5):897-901.

39. Grossman R, Sadetzki S, Spiegelmann R, Ram Z. Haemorrhagic complications and the incidence of asymptomatic bleeding associated with stereotactic brain biopsies. Acta Neurochir (Wien). 2005; Jun 147(6):627-31.

40. Raymond J, Hardy J, Czepko R, Roy D. Arterial injuries in transsphenoidal surgery for pituitary adenoma; the role of angiography and endovascular treatment. AJNR Am J Neuroradiol. 1997; Apr 18(4):655-65.

41. Phatouros CC, Sasaki TY, Higashida RT, Malek AM, Meyers PM, Dowd CF, et al. Stent-supported coil embolization: the treatment of fusiform and wide-neck aneurysms and pseudoaneurysms. Neurosurgery. 2000; Jul 47(1):107-13.

42. Lempert TE, Halbach VV, Higashida RT, Dowd CF, Urwin RW, Balousek PA, et al. Endovascular treatment of pseudoaneurysms with electrolytically detachable coils. AJNR Am J Neuroradiol. 1998; May 19(5):907-11. 